\title{
GENERAL PHYSICAL MODEL FOR SIMULTANEOUS DIFFUSION AND METABOLISM IN BIOLOGICAL MEMBRANES. THE COMPUTATIONAL APPROACH FOR THE STEADY-STATE CASE
}

\author{
JEFFREY L. FOX, CHENG-DER YU, WILLIAM I. HIGUCHI and NORMAN F.H. HO
}

College of Pharmacy, The University of Michigan, Ann Arbor, Mich. 48109 (U.S.A.)

(Final revised version received May 3rd, 1978)

(Accepted May 3rd, 1978)

SUMMARY

A computational approach has been presented for modeling simultaneous diffusion and metabolism in biological membranes. This approach has a virtually unlimited flexibility in the variety and complexity of metabolic schemes that can be modeled. Furthermore, non-uniform enzyme distributions, as well as composite membranes with any number of layers, can also be handled. Example calculations have been presented (using a real system currently being investigated in the author's laboratory) which show that the versatility of this approach is a necessity for the quantitative study of the system. Furthermore, the utility of this approach in guiding decisions as to what path to take in improving a prodrug for a given system is amply illustrated. It is expected that this physical modeling approach, when combined with techniques now being developed for determining the values of the various medel parameters, will provide a very useful tool in the investigation and optimization of drug delivery systems.

\section{INTRODUCTIOIN}

The ability to quantitatively study the simultaneous diffusion and membrane metabolism of drugs is a prerequisite to rational dosage form design. Unfortunately, analytic solutions to the equations used to model these processes are available only for a very few simple cases. In this paper, we will present a computational approach that is applicable to the more complex cases which arise regularly in real biological systems.

\section{BACKGROUND}

The development of quantitative predictive models for drug permeations was initiat d by Scheupleir and co-workers (Scheuplein, 1965, 1967; Scheuplein et al., 1969). For the 
first time the skin barrier was considered in terms of the fractional areas and absolute thicknesses of its strata and other parts. Diffusivities and partition coefficients were recognized as the impo-tant flux-determining properties of a given compound, and the structure dependence of these properties within a simple homologous series (the $n$-alkanols) was also noted.

The concept introduced in the Scheuplein model were used as the basis for a more advanced model in which the effects of $\mathrm{pH}$ were also included in studies of the absorption of various series of ionizable species by human buccal tissue and rat intestinal tissue (Suzuki et al., 1970a, b; Ho and Higuchi, 1971; Ho et al., 1972). More recently, models have evolved which consider the simultaneous transport and metabolisin of drugs by various biomembranes (Rogers, 1975; Ando et al., 1977; Park, 1977; Ho et al., 1976). $\mathrm{Yu}$ et al. (1977) has recently presented a simple model for a more complex chemical system, namely one in which prodrug is converted to the active drug, which in turn is converted to an inactive metabolite. This paper also describes how values for the various parameters in the model could be experimentally determined.

The present paper will describe the numerical methods used to solve much more general models than any mentioned above. The determination of parameter values for these more general models demands a combination of new theoretical techniques, sometimes coupled with new experimental methods, both of which will be described in future publications.

\section{MEMBRANE DIFFUSION AND METABOLISM: THE GENERAL CASE}

This paper will be restricted to the case where the system under study is assumed to be in a steady-state. Even so, the only interesting case where analytical solutions to the system are readily obtainable is when the metabolism obeys first-order kinetics and the responsible enzyme is uniformly distributed throughout the membrane. Ando and Kirschner (1976) showed that an analytical solution was also obtainable for the case where first-order kinetics was obeyed and the enzyme level was directly proportional to the position coordinate in the membrane, but the solution to this restricted case involved fractional order Bessel functions and would therefore be non-trivial to evaluate.

The present approach places no restrictions whatsoever on enzyme activity profiles (other than that they be at least piecewise continuous functions). In addition, the kinetic rate law describing the metabolism could be simple first-order or could involve MichaelisMenten kinetics or competitive, non-competitive, or uncompetitive inhibition. The approach can handle composite membranes, where diffusivities and metabolic properties differ from layer to layer. In addition, systems involving several components can be handled as readily as single component systems.

\section{An example system: prodnig-dnig-metabolite transport through skin}

For purposes of illustration, we will consider a single example system that illustrates many of the complexities found in real systems that our approach is capable of handling. We will consider a relatively simple model in which the skin is composed of two regions, the stratum corneum and the dermis. The system will have three chemical components of 
interest, which we will denote as prodrug, drug, and metabolite. This is representative of a real system in which a relatively non-active compound (a prodrug) gets metabolized $10 \mathrm{a}$ relatively active compound (drug), which in turn is metabolized to another inacive convpound (metabolite). We shall assume that uoth metabclic steps obey Michaelis-Menten kinetics, and furthermore that the drug to metabolite step can be competitively inhibited by the prodrug as well as by an inhibitor that coul: be added to the system. The metabolic scheme is in accordance with one currently being investigated in cout tabotatories for the transport and metabolism of prodrugs of 9-3-D-arabinofuranowylatentine (ara-A). Lipper (1978) has shown this scheme to be operable in both in witro erayme kinetic studies and in cell suspension studies of the transport and metabolisnt of araand a prodrug, ara-A-S'-valerate.

\section{Mathematical description of the model}

The model for the example system we shall use in this paper is shown in Fig. 1 . For convenience the stratum corneum and dermis will be referred to as the left and tight regions of the model and their thickness denoted by $L$ and $R$. Concentrations of prodnes. drug, and metabolite will be denoted by $P, D$, and $M$. Diffusivities of the species in the two regions of the model will be symbolized by DSQ where $S$ represents the species natue and $Q$ the region. For example, DDR represents the Diffusivity of the Drug irn the Right. hand region (the dermis). The activities of the enzymes in the dermis responsible for the prodrag to drug and drug to metabolite conversions will be denoted by $\mathrm{El}$ and $\mathrm{E} 2$. (Recall that these may vary with position coordinate.) In the example calculations it is assumed that there is no enzyme activity in the stratum corneum.

The differential equations describing the simultaneous diffusion and metabolism in the dermal region are then developed as follows. The rate of enzymatic conversion of prodrug to drug (assuming Michaelis-Menten kinetics) is given by:

$\frac{V_{\max 1}[\mathrm{P}]}{\mathrm{K}_{\mathrm{ml}}+[\mathrm{P}]}$

CORNEUN DERMIS

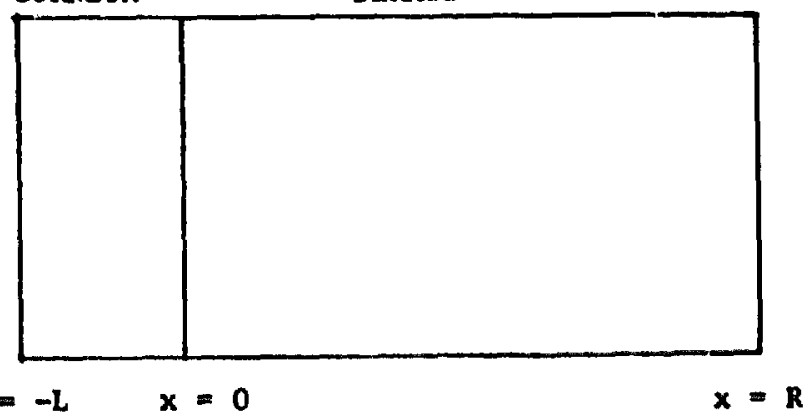

Fig. 1. Schematic of two layer skin model used in example calculation. 
and the rate of conversion of drug to metabolite is given by:

$\frac{\mathrm{V}_{\max 2}[\mathrm{D}]}{\mathrm{k}_{\mathrm{m} 2}\left(1+\frac{[\mathrm{P}]}{\mathrm{KI}}+\frac{[\mathrm{I}]}{\mathrm{K}_{\text {inhib }}}\right)+[\mathrm{D}]}$

where this metabolic step is assumed to be competitively in hibited by both prodrug and another inhibitor, I, which could be added to the system. (In some of our experiments with ara-A, coviderabine has been used in this capacity.)

The differential equation describing the behavior of prodrug is obtained by combining the diffusion equation (Fick's second law) with the first term above for prodrug metabolism to give

$\frac{\partial[P]}{\partial t}=D P R \frac{\partial^{2}[P]}{\partial_{x^{2}}}-\frac{V_{m a x l}[P]}{K_{m 1}+[P]}$

Assuming steady-state (i.e. $\partial[\mathrm{P}] / \partial \mathrm{t}=0$ ), and omitting the brackets, this becomes

$\operatorname{DPR} \frac{\mathrm{d}^{2} \mathbf{P}}{\mathrm{dx} \mathrm{x}^{2}}-\frac{\mathrm{V}_{\mathrm{maxl}} \cdot \mathbf{P}}{\mathrm{K}_{\mathrm{ml}}+\mathbf{P}}=0$

Similar treatments yield the differential equations for the steady-state behavior of the drug and the metabolite. These equations can then be rewritten as:

$\mathrm{DPR} \cdot \frac{\mathrm{d}^{2} \mathrm{P}}{\mathrm{dx}^{2}}-\frac{\mathrm{El} \cdot \mathrm{P}}{1+\mathrm{KPI} \cdot \mathrm{P}}=0$

$\mathrm{DDR} \cdot \frac{\mathrm{d}^{2} \mathrm{D}}{\mathrm{dx}^{2}}+\frac{\mathrm{El} \cdot \mathrm{P}}{1+\mathrm{KP} 1 \cdot \mathrm{P}}-\frac{\mathrm{E} 2 \cdot \mathrm{D}}{1+\mathrm{KD} \cdot \mathrm{D}+\mathrm{KP} 2 \cdot \mathrm{P}+\mathrm{KI} 2 \cdot \mathrm{I}}=0$

$\mathrm{DMR} \cdot \frac{\mathrm{d}^{2} \mathrm{M}}{\mathrm{dx}^{2}}+\frac{\mathrm{E} 2 \cdot \mathrm{D}}{1+\mathrm{KD} \cdot \mathrm{D}+\mathrm{KP} 2 \cdot \mathrm{P}+\mathrm{KI} 2 \cdot \mathrm{I}}=0$

where

$\mathrm{El}=\mathrm{V}_{\operatorname{maxl}} / \mathrm{K}_{\mathrm{ml}}$

$\mathrm{KPI}=1 / \mathrm{K}_{\mathrm{ml}}$

$\mathrm{E} 2=\mathrm{V}_{\max 2} / \mathrm{K}_{\mathrm{m} 2}$

$\mathrm{KD}=1 / \mathrm{K}_{\mathrm{m} 2}$

$\mathrm{KP} 2=1 / \mathrm{KI}$

$\mathrm{KI} 2=1 / \mathrm{K}_{\text {inhib }}$

The above forms were chosen for mathematical convenience in that an effect can be omitted from the system simply by zeroing the appropriate coefficient. 
Since there are no metabolic processes occurring in the stratum corneum the solutions to the diffusion equations result in linear concentration gradients in this region. These solutions can be combined with the boundary conditions at $x=-L$ and the stipulation that fluxes be continuous to give the following set of boundary conditions for $x=0$ :

$$
\begin{aligned}
& \frac{D P L}{L}(P L-P(0))=-D P R \cdot P^{\prime}(0) \\
& \frac{D D L}{L}(D L-D(0))=-D D R \cdot D^{\prime}(0) \\
& \frac{D M L}{L}(M L-M(0))=-D M R \cdot M^{\prime}(0)
\end{aligned}
$$

where

PL, DL, ML = the given concentrations of prodrug, drug, and metabolite at $\mathrm{x}=-\mathbf{L}$;

$P(0), D(0), M(0) \quad=$ the concentrations at $x=0$

$P^{\prime}(0), D^{\prime}(0), M^{\prime}(0)=$ the gradients in the dermis at $x=0$

At $x=R$, concentrations of the various species are assumed to be zero:

$P(R)=D(R)=M(R)=0$

\section{Computation solution of the model}

Eqns. $1-3$ represent a boundary value problem with mixed boundary conditions at the left-hand side and Dirichlet conditions at the right-hand side. In the general case, for a system such as this, all the equations must be solved simultaneously. Examination of this system, however, reveals that they may be solved in sequence. The prodrug concentration profile is computed first, for it is independent of the other concentrations; then the drug profile, which is dependent only on the prodrug profile; finally the profile for the metabolite is computed.

As the solutions for these equations are obtained in very similar manners, we will describe in detail the solution process for the first one only. This equation represents a non-linear boundary value problem, and thus will necessitate use of an iterative technique in obtaining its solution. Although problems of this type may be solved by using either finite difference or finite element techniques, the use of either of these families of techniques requires considerable developmental time just to ensure that the chosen method is both stable and sufficiently accurate for the problem being solved. Furthermore, the stability and accuracy characteristics of a chosen scheme might change if the model were modified. For these reasons we wanted to use a technique which could incorporate an already developed software component (in this case an ordinary differential equation solver) capable of controlling the accuracy of the calculation and also of minimizing the smount of work necessary to achieve this accuracy. Finally, it was desirable that the 
technique be such that changes in the model could be easily incorporated.

The method chosen was the method of adjoints, one of a family of techniques known as 'shooting methods', several of which are mathematically equivalent although formulated quite differently (Shipman and Roberts, 1972). The general scheme behind the various shooting methods is the following: a set of initial values for the problem is chosen that is consistent with the boundary conditions for the left-hand side; the system is integrated from left to right; if the result of this integration satisfies the right-hand boundary condition, the problem is solved; if not, a different set of iritial conditions is chosen and the procedure repeated. The method of adjoints provides a means of choosing the initial conditions for repeating the integration.

Implicit in this scheme is the assumed ability to accurately integrate the nonlinear equation(s). We have chosen an integrator written by Gear (1971) which is capable of solving 'stiff' systems of equations, which are systems where greatly differing coefficients arising in the equations can effectively prohibit accurate solution via more general numerisal techniques. Unfortunately, this technique requires more information than conventional techniques. For a system of equations written as

$y^{\prime}=g(y)$

stiff methods require the ability to compute the matrix of partial derivatives

$\frac{\partial g}{\partial y}$

in addition to the ability to compute $y^{\prime}$. Even so, this is a small price to pay for the assurance that a method is 'robust' in the sense that most pl. vsically reasonable problems are handled without difficulty.

The solution of Eqn. 4 by the method of adjoints is as follows. Redefine the equation in terms of normalized variables and rewrite is as a system of first-oruer equations:

$t=\frac{x}{R}$

$y_{1}=\frac{P}{P L} \cdot f \quad$ where $f=1+\frac{D P R \cdot L}{D P L \cdot R}$

$\mathrm{y}_{2}=\frac{\mathrm{dy}}{\mathrm{dt}}$

$\frac{d y_{2}}{d t}=\frac{d^{2} y_{1}}{d t}=\frac{R^{2} \cdot E l / D P R \cdot y_{1}}{1+K P I \cdot P L \cdot y_{1} / f}$

or $\mathrm{y}^{\prime}=\mathrm{Uy}$

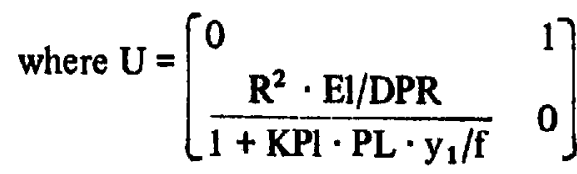


The boundary conditions become

$$
\begin{aligned}
& \mathrm{q}_{1} \equiv 1-\mathrm{y}_{1}(0)+\frac{\mathrm{DPR} \cdot \mathrm{L}}{\mathrm{DPL} \cdot \mathrm{R}}\left(1+\mathrm{y}_{2}(0)\right)=0 \\
& \mathrm{q}_{2} \equiv \mathrm{y}_{1}(1)=0
\end{aligned}
$$

The matrix of partials needed by the integrator is:

$$
\frac{\partial g}{\partial y}=\left[\begin{array}{ll}
0 & 1 \\
\frac{R^{2} \cdot E l / D P R}{\left(1+K P l \cdot P L \cdot y_{1} / f\right)^{2}} & 0
\end{array}\right]
$$

This system is integrated from $t=0$ to $t=1$ with the $y_{1}$ profile being saved. Then $q_{1}$ and $q_{2}$ are calculated, and if less than some preset tolerance, the problem is considered solved. Otherwise, it is necessary to integrate the system of adjoint equations:

$\mathrm{x}^{\prime}=-\mathrm{U}^{\mathrm{T}} \mathrm{x}$

from $x=1$ to $x=0$ with the initial conditions

$x(1)=\left(\begin{array}{l}1 \\ 0\end{array}\right)$

where $-U^{T}$ is the negative transpose of $U$. As $U$ is not a function of $x$, the matrix of partials is also given by $-U^{T}$. In performing this integration it is necessary to be able to compute $E 1$ and $y_{1}$ for given values of $t$. For this reason the $y_{1}$ profile was saved as it was formed and values of $y_{1}$ for a given value of $t$ then obtained using a spline interpolai in technique.

The new initial values for $y$ are then calculated as follows. Compute $\delta y(0)$ from

$$
\left[\begin{array}{ll}
-1 & \frac{\mathrm{DPR} \cdot \mathrm{L}}{\mathrm{DPL} \cdot \mathrm{R}} \\
\mathrm{x}_{1}(0) & \mathrm{x}_{2}(0)
\end{array}\right]\left[\begin{array}{l}
\delta \mathrm{y}_{1}(0) \\
\delta \mathrm{y}_{2}(0)
\end{array}\right]=\left[\begin{array}{l}
-\mathrm{q}_{1} \\
-\mathrm{q}_{2}
\end{array}\right]
$$

where the first row of the matrix is the first row of $\partial q / \partial y$. Then set

$y(0)=y(0)+\delta y(0)$

and begin the process again. The method of adjoints is capable of handling any combination of Dirichlet, Neumann, or mixed boundary conditions. This problem is a simple example of the more general problem, which is described elsewhere (Shipman and Roberts, 1972).

\section{Program organization}

The overall organization of the computer program used for calculations involving the diffusion metabolism model is shown in Fig. 2. The hierarchy of the various numerical routines is dictated by the nature of the technique being used. It should be noted, how. ever, that many of the lower level routines have multiple entry points, one entry being 


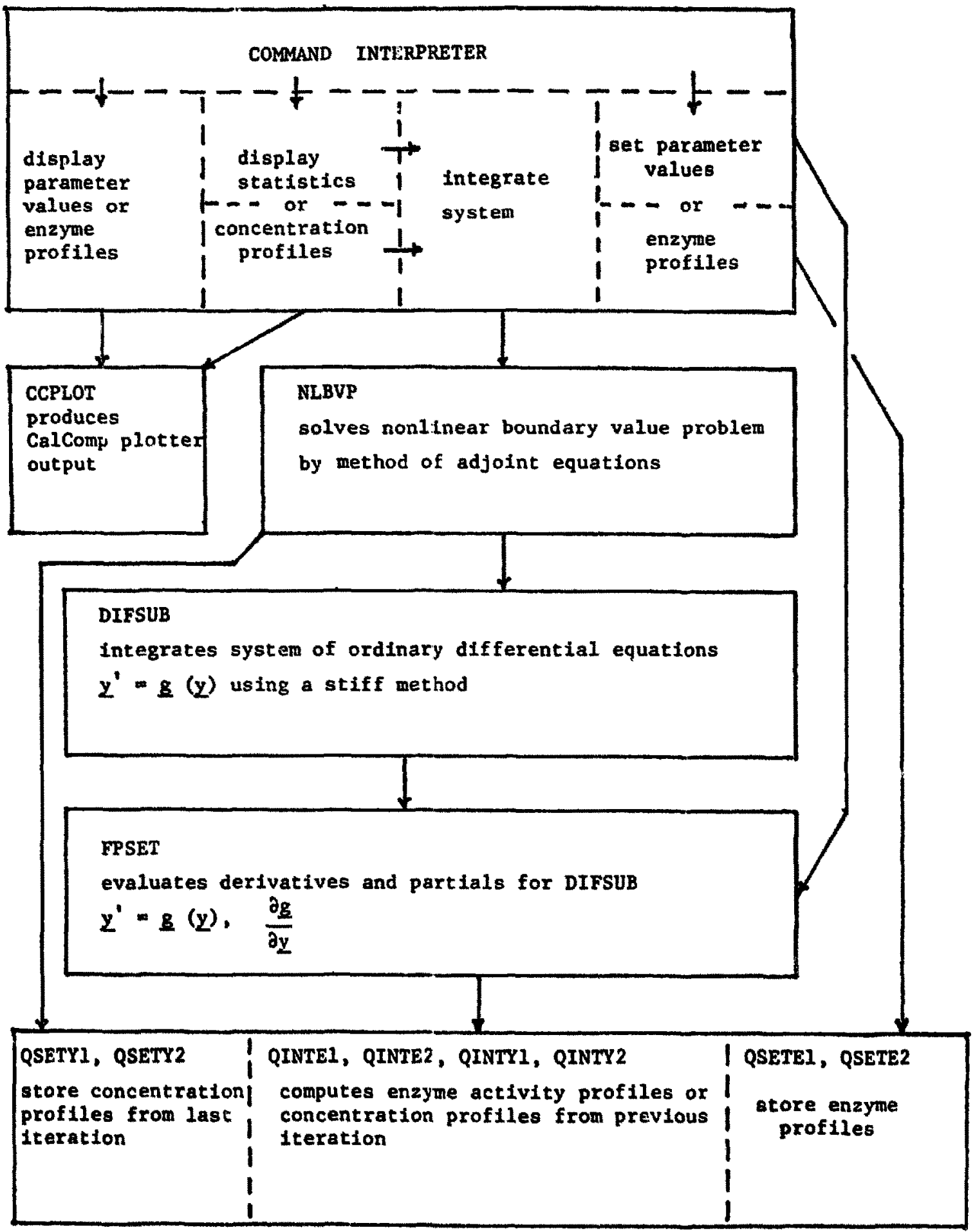

Fig. 2. Organizational structure of computer program to solve steady-state prodrug-drug-metabolite simultaneous diffusion and metabolism problem. 
used only on occasion to set values of parameters within the routine, white the othet entry points are used as needed by higher level routines. A scheme such as this greatly facilitates writing and making changes to the program becuase the passing of parametert among routines is greatly ninimized. Unfortunately this scheme, while efficient in terms of both program divelopment time and actual program execution time, apparently is not compatible witl: the FORTRAN dialects used by all compilers. For comphe encounter no difficulties when using the IBM level $G$ compiler; the IBM level $I I$ compater, on the other hand, produces code that does not execute properiy.

The main program is written in FLECS (Beyer, 1974\%, a language extension of FORTRAN which has several additional mechanisms for controlling program now. We have found FLECS to be of tremendous value when writing programs which have multitude of possible program flow patterns.

\section{Example calculations and applications to drug design}

Some example calculations will be presented in order to illusirate the capubiligy ou: computationa! approach has for handling quite complex diffusion-metabolism problems. and also to show that this capability is indeed essential for the systems we are studying.

\section{TABLE 1}

\section{PARAMETER VALUES USED IN CALCULATIONS}

\begin{tabular}{|c|c|c|}
\hline Parameter & Description & Value \\
\hline $\begin{array}{l}\mathbf{L} \\
\mathbf{R}\end{array}$ & $\begin{array}{l}\text { stratum corneum thickness } \\
\text { dermal thickness }\end{array}$ & $\begin{array}{l}4 \times 10^{-3} \mathrm{~cm} \\
3 \times 10^{-2} \mathrm{~cm}\end{array}$ \\
\hline $\mathbf{P L}$ & $\begin{array}{l}\text { concentration of prodrug on left-hand side (assumed } \\
\text { equal to prodrug solubitlity) }\end{array}$ & $2 \times 10^{-2} \operatorname{mmoth}$ \\
\hline DL, ML & concentration of drug and metabolite on left-hand side & 0 \\
\hline $\begin{array}{l}\text { DPL } \\
\text { DDL } \\
\text { DML }\end{array}$ & $\begin{array}{l}\text { diffusivities of prodrug } \\
\text { drug and metabolite } \\
\text { in stratum corneum }\end{array}$ & $\begin{array}{l}2.4 \times 10^{-10} \mathrm{~cm}^{2} / \mathrm{csc}^{-1} \\
8.0 \times 10^{-11} \mathrm{~cm}^{2} / \mathrm{sec}^{-6} \\
8.0 \times 10^{-11} \mathrm{~cm}^{2} / \mathrm{sec}\end{array}$ \\
\hline $\begin{array}{l}\text { DPR } \\
\text { DDR } \\
\text { DMR }\end{array}$ & $\begin{array}{l}\text { diffusivities of prodrug } \\
\text { drug, and metabolite } \\
\text { in dermis }\end{array}$ & $\begin{array}{l}3.7 \times 10^{-8} \mathrm{~cm}^{2} / \mathrm{wc} \\
3.8 \times 10^{-9} \mathrm{~cm}^{2} / 4 \mathrm{sec} \\
3.8 \times 10^{-7} \mathrm{~cm}^{2} / \mathrm{sec}^{2}\end{array}$ \\
\hline $\begin{array}{l}\mathrm{KP1} \\
\mathrm{KD} \\
\mathrm{KP2} \\
\mathrm{KI} 2\end{array}$ & $\begin{array}{l}1 / K_{\mathrm{ml}} \text { (esterase) } \\
1 / \mathrm{K}_{\mathrm{m2}} \text { (deaminase) } \\
1 / \mathrm{K} \mathbf{I} \text { (deaminase) } \\
1 / \mathrm{K}_{\mathrm{inhib}} \text { (deaminase) }\end{array}$ & $\begin{array}{l}1000 \mathrm{~cm}^{3} \text { mol } \\
1 \times 10^{4} \mathrm{~cm}^{3} \text { monol } \\
1 \times 10^{5} \mathrm{~cm}^{3} \text { mowot } \\
0\end{array}$ \\
\hline $\begin{array}{l}\text { E1MAX } \\
\text { E2MAX }\end{array}$ & $\begin{array}{l}\text { maximum value of } V_{\max } / K_{m 1} \text { (esterase) } \\
\text { maximum value of } V_{\max 2} / K_{m 2}\end{array}$ & $\begin{array}{l}3 \times 10^{-3} 3^{-1-13} \\
4 \times 10^{-3} n^{-1}\end{array}$ \\
\hline
\end{tabular}

a This parameter was varied in some studies; otherwise the indicated value was used. 


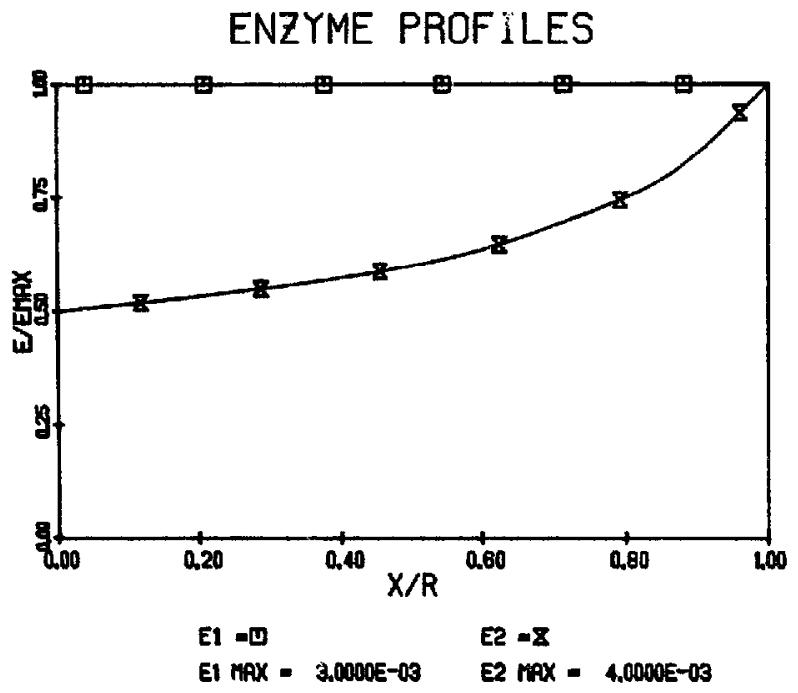

Fig. 3. Normalized enzyme activity profiles used in example calculation: E1, esterase; E2, deaminase.

Furthermore, the possibility for the direct application of these calculations to rational drug design will be illustrated.

Using reasonable estimates for values of the various parameters (see Table 1), the concentration profiles resulting from the steady-state transport and metabolism of ara-A-5'valerate from a saturated aqueous solution into and through the hairless mouse skin were calculated. Ara-A-5'-valerate (the inactive prodrug) is de-esterified to form ara-A (thought to be an active antiviral), which is in turn deaminated to the inactive ara-hypoxanthine (ara-H), this latter step being competitively inhibited by the prodrug. This system is modelled by Eqns. 1a-c. In examining the results of the calculation, it should be borne in mind that the parameter values used were only estimates; for example, the dermal enzyme activity profiles shown (normalized) in Fig. 3 were used (since the actual profiles have not been resolved yet), and the saturability of deaminase by ara-A and its competitive inhibition by the prodrug were assumed to quantitatively correspond to results obtained from beaker experiments ${ }^{*}$ with a commercially available deaminase preparation. Nonetheless, the example calculations should provide a good qualitative idea of what might later be expected from calculations using more accurately known parameter values.

The calculated normalized dermal concentration profiles of ara-A-5'-valerate, ara-A, and ara-H (denoted by $P, D$, and $M$, respectiveiy) are shown in Fig. 4. The calculated ara-A level at the left-hand side of the dermal region is at about (or slightly above) the level thought to be necessary for antiviral activity in vero renal tissue culture (Williams and Lerner, 1975). This is indicative of the possibility that the $5^{\prime}$-valerate ester might be almust good enough to be an effective antiviral agent in the hairless mouse skin. The fact that the concentration profiles for the three species are all at comparable levels means that any change in the parameters describing the metabolic processes would directly affect the calculated profiles. Also of note is that the calculated level of 5 -valerate is such that the ara-A to ara-H rate of conversion is reduced between $50 \%$ and $75 \%$ through 
CONCENTRATION PROF ILES

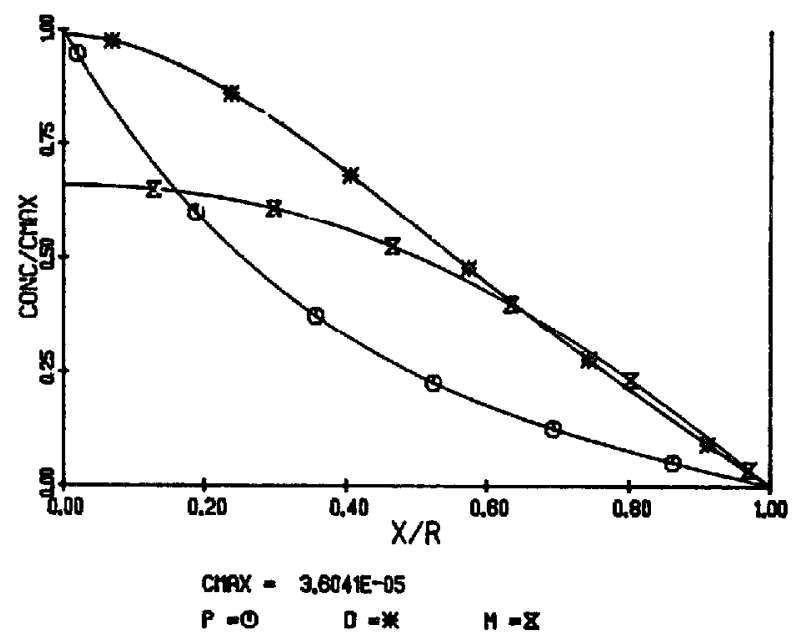

Fig. 4. Normalized concentration profiles in dermis in example calculation: $\mathrm{P}$, prodrug (ara-A-5" valerate); D, drug (ara-A); M, metabolite (ara-H).

approximately the first half of the dermal region as a result of the competitive inhibition of this step by the prodrug.

The results of the above calculation show the futility of attempting prodrug design in this system without the capability of quantitatively accounting for changes in each of the relevant parameters. For the system shown in this calculation there are no fewer than four parameters that must be considered in assessing the effect of modifying the prodrug. These parameters are:

(1) Solubility of the prodrug

(2) Permeability of the prodrug (especially to the stratum corneum, but also to the dermis)

(3) Metabolism of the prodrug ara-A

(4) Ability to competitively inhibit the ara-A to ara-H conversion.

The effects of changing the values of these parameters have been assessed in a series of calculations which are summarized in Figs. 5-10. In these calculations we vary each parameter separately, while holding the others constant, even though any change in the structure of the prodrug is apt to change all of these parameters. We are currently studying the $5^{\prime}$-acetate and $5^{\prime}$-octanoate esters of ara-A as well as the $5^{\prime}$-valerate in order to begin to see how the various parameters depend on molecular structure. As a measure of activity we will use the area under the dermal concentration profile curve. This quantity has units of $\mathrm{mmol} / \mathrm{cm}^{2}$ and corresponds to the amount of ara-A per unit area of dermis. In our calculations we will study the effects of changes in prodrug solubility, rate of prodrug conversion to drug, and in ability of the prodrug to inhibit the metabolism of drug to metabolite.

Fig. 5 shows the effect of prodrug solubility on ara-A activity. The very slight deviations of the curve from the strict linearity seen at the low end are a result of beginning to saturate the deaminase (responsible sor the drug to metabolite conversion) in the 
LOG10 (ARA-A/CM-SQ)

vS.

LOG 10 (FRODRUG SOLUBILITY)

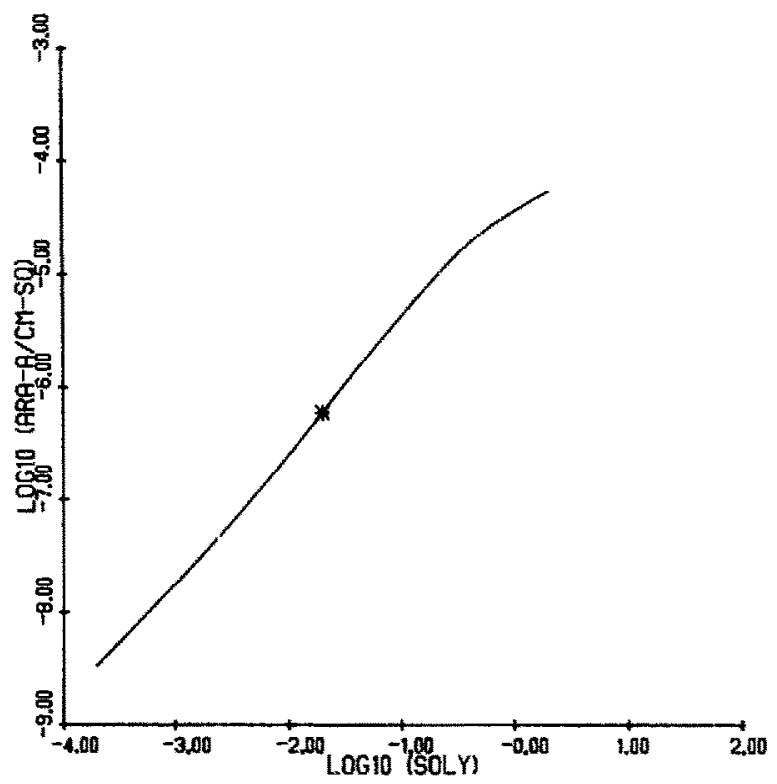

Fig. 5. Dependence of ara-A activity on prodrug solubility. Model calculation for intact hairless mouse skin. (* indicates ara-A-5'-valerate.)

$$
\text { ARA-A/CM-SO }
$$

vS.

$$
\text { LOG10 (KI) }
$$

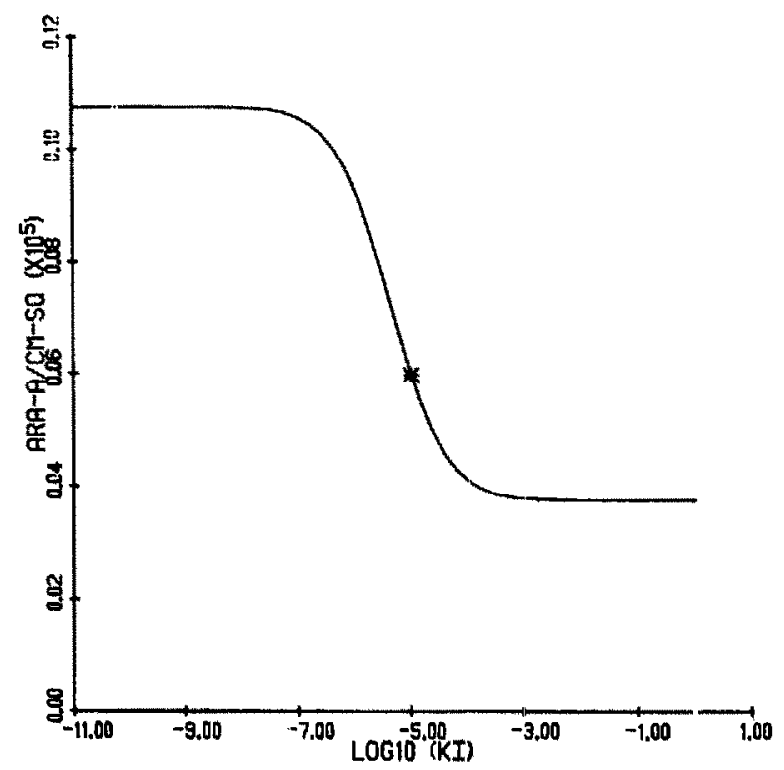

Fig. 6. Dependence of ara-A activity on the ability of prodrug to inhibit deamination of ara-A. Model calculation for intact hairless mouse skin. (* indicates ara-A-5'-valerate.) 
- VS.

\section{LOC10 (VMAX1/KM1)}



Fig. 7. Dependence of ara-A activity on the lability of prodrug to esterase. Model calculation for intact hairless mouse skin. ( ${ }^{*}$ indicates ara-A-5'-valerate.)

middle of the curve and the esterase (responsible for the prodrug to drug conversion) at the top end of the curve. The star in the middle indicates where the 5 -valerate falls on the curve and shows that for this system an increase or decrease in prodrug solubility will result in an approximately proportionate change in ara-A activity.

Fig. 6 shows the dependence of ara-A activity on the ability of the prodrug to inhibit the drug to metabolite conversion. This is an extremely valuable plot for the would be designer of an ara-A prodrug. It shows that even if the prodrug were to completely inhibit this conversion, ara-A levels would increase only by about two-fold, whereas if there were no inhibition whatsoever, the ara-A level would decrease by only about $40 \%$. This is somewhat surprising in light of the fact that in cell suspension studies, the prodrug inhibition of drug metabolism results in a many-fold increase in intercellular drug levels. Furthermore, a deaminase inhibitor (covidaribine) increases the intracellular ara-A levels by several orders of magnitude in cell suspensions. As Fig. 6 shows, any attempt to extrapolate this tremendous enhancement to the intact hairless mouse skin situation will be met with disappointment, as only about a two-fold increase will occur. This result underscores both the importance of appropriate physical modeling to understanding the systems we work with and also the dangers of measuring drug activity in some laboratory system and attempting to extrapolate this result to another biological system withnut appropriate accounting for geometric as well as biochemical differences.

Fig. 7 shows the result of another possible prodrug modification, the enhancement of the lability of prodrug to cleavage by esterase. A naive approach would assume that 
increasing the lability of prodrug would give a concomitant increase in drug activity. As Fig. 7 shows, this is not the case at all; in fact, increasing the lability will antually cause a slight decrease in drug activity. This is a result of two factors: the lability is already sufficiently high to keep up with the prodrug diffusion through the stratum corneum; as lability increases the penetration through the stratum corneum becomes rate limiting and the curve flattens out. In addition, prodrug is also serving as an inhibitor of drug metabolism; thus as lability increases and prodrug is more rapidly removed, the drug is more readily metabolized, resulting in an actual decrease in drug activity. Hence there is an optimum prodrug lability in this system, as Fig. 7 shows, the $5^{\prime}$-valerate is positioned almost exactly at this optimum.

The net conclusion of Fig. 5-7 is that any improvements in the prodrug designed to enhance ara-A levels in the intact skin of the test animal will be in the area of enhancing solubility. Other possible modifications such as increasing prodrug lability to esterase or decreasing the rate of drug deamination are simply not effective in this system. As we shall see in Figs. 8-10, however, if the stratum corneum is removed, our strategy for prodrug improvement will be quite different.

The problem of maximizing ara-A levels in the special case where no stratum corneum is present is of more than academic interest. For example, ara-A might be applied therepeutically either to skin where the stratum corneum is absent as a result of lesion formation or has been removed as part of the treatment or to vaginal membranes where no

LOG10 (ARA-A/CM-SQ)

VS.



Fig. 8. Wependence of ara-A activity on prodrug solubility. Model calculation for hairless mouse skin with stratum corneum removed. ( ${ }^{*}$ indicates ara-A-S'-valerate.) 
stratum corneum is present in the first place. We do not wish to imply that vaginal membranes are quantitatively or even qualitatively similar to dermis as regards the fat of ara-A and its prodrugs since experiments to assess this have not yet been done. We cite the example of the vaginal membrane simply to point out the importance of the case of ara-A transport and metabolism in a membrane without a diffusional barrier in front of it.

Fig. 8 shows the denenderice of ara-A activity on predrug solubility when everything in the system is the sanie as the previous examples, except that no stratum corneum is present. The first point worthy of nots is that the ara-A activity is about 25 times higher than when the scratum corneum was presert. Second (and somewhat surprising), increasing the prodrig solubility will not improve the ara-A level, although a decreased prodrug solubility would lead to a decreased ara-A level. This indicates that the prodrug solubility is already sufficiently high and that some other factor is limiting the attainable ara-A level.

Fig. 9 shows the dependence of ara-A activity on the ability of prodrug to inhibit the ara-A to ara-H deamination. This factor, which was relatively unimportant in the case where stratum corneum was present, now has almost no effect whatsoever. This is because the ara-A level is now high enough to approach saturation of the deaminase so that deamination only removes a small fraction of the ara-A. Therefore, additional inhibition of this deamination has a very small overall effect.

LOG10 (ARA-A $/ C M-S Q)$

VS.

ARA-A/CM-SO

vs.

LOG1O (KI)
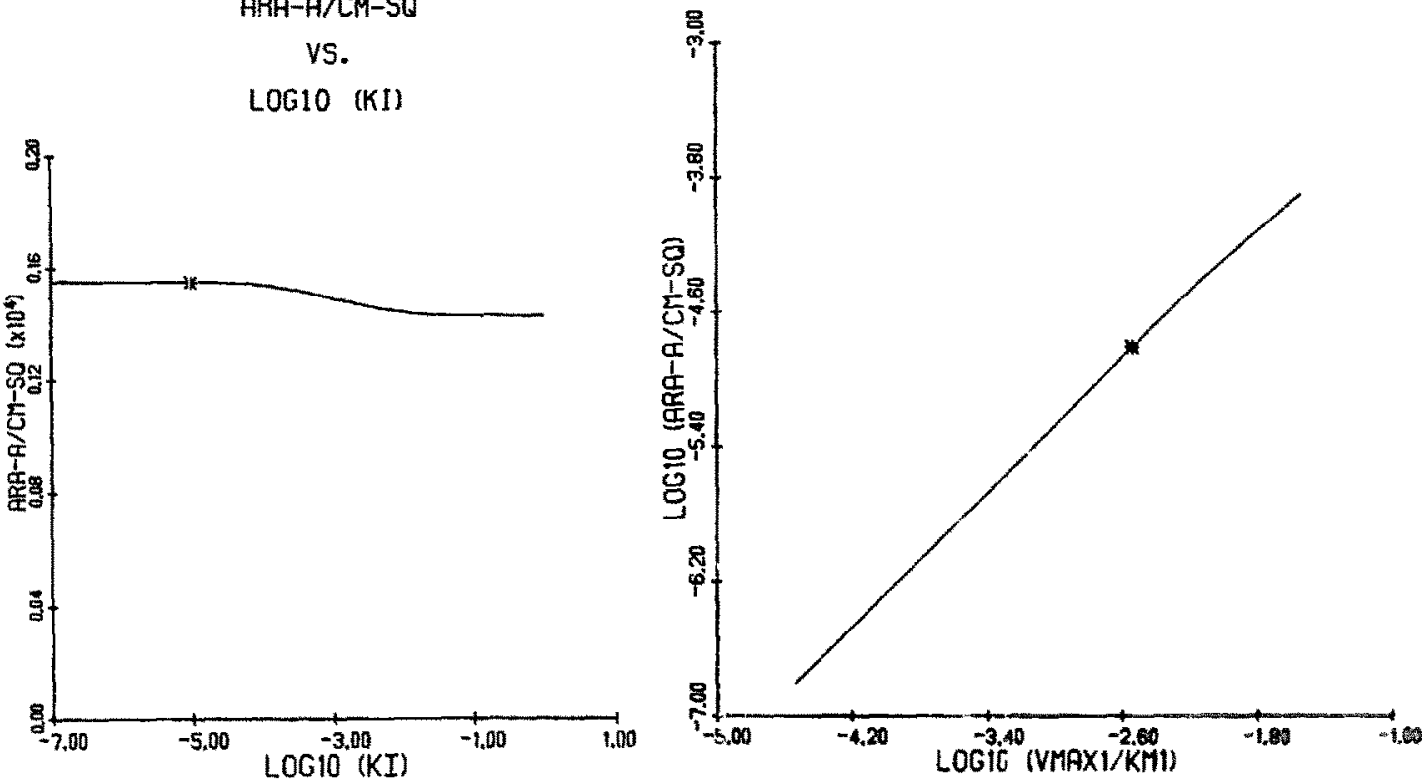

Fig. 9. Dependence of ara-A activity on the ability of prodrug to inhibit deamitiation of ara-A. Model calculation for hairless mouse skin with stratum co: neum removed. (*indicates ara-A-5'-valerate.)

Fig. 10. Dependence of ara-A activity on the lability of prodrug to esterase. Model calculation for hairless mouse skin with stratum corneum removed. (* indicates ara-A-5*-valerate.) 
The dependence of ara-A activity on the rate of the prodrug to ara-A conversion is shown in Fig. 10. This dependence is substantially linear and indicates that an increase in prodrug lability to esterase will result in a proportionate increase in ara-A activity in the case where stratum corneum is absent. Hence the ara-A level in this system is limited by esterase lability, wherease when stratum comeum was present, prodrug solubility was the limiting factor ${ }^{1}$.

\section{DISCUSSION}

The foregoing calculations should make apparent the value of detailed physical modeling to the rational design of prodrugs. Three different factors (prodrug solubility, rate of prodrug to drug conversion, and ability of prodrug to inhibit the metabolism of the drug) were considered and their relative importances were seen to depend on the sysiem being studied. In the intact skin model, ara-A activity could be improved significantly only by increasing prodrug solubility, whereas in the model with no stratum corneum barrier, the ara-A level could be increased only by increasing the susceptibility of prodrug $\mathrm{t} J$ esterase. In neither of these situations was the deamination of ara-A an important factor. In the case of cell suspensions, however, it has teen shown that deamination is important and its inhibition can lead to a many-fold increase in intracellular ara-A levels. These conclusions are based on the assumption that in each of these systems ara-A and its prodrugs undergo the same metabolic processes. The differences in behavior of these model systems are therefore primarily a function of the different geometries and the permeabilities of species in the various regions of these systems.

This suggests a new systematic approach to the rational design of prodrugs. Emphasis woulc be placed on the quantitative description of the relevant transport and metabolism processes in the target tissue. The use of animal or in vitro systems would be directed toward the study of transport and metabolism for a family of prodrugs, with the hope that the measured dependencies of these parameters on molecular structure would also be applicable to the target organism. Thus, finding model enzyme or cell systems capable of mimicing the metabolic fate of the prodrug in the target system would be of primary consideration, regardless of the effectiveness of the drug in eliciting a pharmacological response in the model system. Developed sufficiently, this detailed physical modeling approach should lead directly to the selection of the optimal prodrug from a given fanily of prodrugs for delivery to a specified site of action.

\section{REFERENCES}

Ando, H.Y., Ho, N.F.H., Higuchi, W.I., Turi, J. and Shipman, C., Jr., Systems approach to the study of drug transport across membranes using suspension cultures of mamalian cells VI: simultancous passive transport and biosynthesis. J. Theoret. Biol., 62 (1976) $211-225$.

\footnotetext{
${ }^{1}$ It should be noted that the $K_{m}$ for esterase was not measured, but for purposes of calculation arbitrarily assigned a value of $10^{-3} \mathrm{mmol} / \mathrm{cm}^{3}$. The ratio $V_{\max } / \mathrm{K}_{\mathrm{m}}$ for esterase was measured experimentally in the hairless mouse skin. The main point of these calculations, namely the importance of a complete a modeling job as possible, is in no way lessened by uncertainties in any of the parameter values for illustrative calculations.
} 
Ando, H.Y. and Kirschner, A.S., Drug transport across a model epidermus with a linear distribution of metabolizing enzyme. Manuscript in preparation.

Boyer, T., FLECS: User's Manual. Department of Computer Science, University of Oregon, Eugene, Oregon, 1974.

Gear, C.W., Numerical Initial Value Problems in Ordinary Differential Equations, Prentice-Hafl, Englewood Cliffs, N.J., 1971.

Ho, N.F.H. and Higuchi, W.I., Quantitative interpretation of in vivo buccal absorption of $n$-alkanoic acids by the physcial model approach. J. Pharm. Sci., 60 (1971) 537-541.

Ho, N.F.H., Higuchi, W.I. and Turi, J., Theoretical model studies of drug alssorption and transport in the GI tract III. J. Pharm. Sci., $61(19 \% 2) 192-197$.

Ho, N.F.H., Park, J., Morozowich, W. and Higuchi, W.I., Physical niod sl approach to the design of drugs with improved intestinal absorption. In E.B. Roche, (Ed.), Dfsign of Biopharmaceutical Properties Through Prodrugs and Analogs, American Pharmaceuti a Association, Academy of Pharm. Sci., Washington, D.C., 1977, pp. 98-185.

Lipper, R.A., Ph.D. Thesis. The University of Michigar, 1978.

Park, J.Y., Ph.D. Thesis. The University of Michigan, $197 \%$.

Rogers, J.D., Ph.D. Thesis. The University of Michigan, 1976.

Scheuplein, R.J., Mechanism of percutaneous absorption I: routes of pinetration and the influence of solubility. J. Invest. Dermi., 45 (1965) 334-34t.

Scheuplein, R.J., Mechanism of percutaneous absorption II: transient diffusion and the relative importance of various routes of skin penetration. J. Invest. Derm., 48 (1967) 79-88.

Scheuplein, R.J., Blank, I.H., Brauner, G.J. and MacFarlane, D.J., Percuta teous absorption of steroids. J. Invest. Derm., 52 (1969) 63-70.

Shipman, J.S. and Roberts, S.M., Two-Point Boundary Value Problems: Shooting Methods, American Elsevier, New York, 1972.

Suzuki, A., Higuchi, W.I. and Ho, N.F.H., Theoretical studies of drug ab iorption and transport in the gastrointestinal tract 1. J. Pharm. Sci., 59 (1970a) 644-651.

Suzuki, A., Higuchi, W.I. and Ho, N.F.H., Theoretical studies of drug absorption and transport in the gastrointestinal tract II. J. Pharm. Sci., 59 (1970b) 651-659.

Williams, B. and Lerner, A.M., Minimal inhibitory concentrations and minimal virucidal concentrations of 9- $\beta$-D-arabinofurasonyladenine and 9- $\beta$-D-arabinofuranosylhypoxanthine with and withe an inhibitor (Warner-Lambert/Parke-Davis) of adenosine deaminase vs herpes simplex virsu, type I. In Antivirals with Clinica: Potential, A Symposium at Stanford University, Stanford, Calif., August 26-29, 1975.

Yu, C.D., Fox, J.L., Higuchi, W.I. and Ho, N.F.H., Physical model approach to the topical delivery of a prodrug in skin. Initial studies of the simultaneous transport and bioconversion of a 5 ester of adenine- $\beta$-D-arabinofuranoside (Ara-A). J. Pharm. Sci., submitted for publication, 1978. 